\title{
Mobile Robots for Difficult Measurements in Difficult Environments: Application to Aging Aircraft Inspection
}

\author{
M. W. SIEGEL \\ Robotics Institute \\ W. M. KAUFMAN and C.J. ALBERTS \\ Carnegie Mellon Research Institute \\ Carnegie Mellon University \\ Pittsburgh PA 15213 USA
}

\begin{abstract}
This paper describes a systematic approach to using robots to automate making difficult measurements in real-world environments. The approach is illustrated by a detailed description of the architecture's actual application to inspection of commercial aircraft for skin cracks and lap joint delamination. Several robot designs are evaluated; a small mobile robot with suction cup feet is found to be best in the practical context of commercial aircraft maintenance and inspection. Approaches are discussed for primary eddy-current flaw detection sensor signal understanding and for secondary vision-based guidance image understanding, The prospects for vision-based inspection are discussed.
\end{abstract}

\section{Background}

We examined numerous technologies that use sensors to detect concealed flaws and materials, e.g., cracks in aircraft skin [1], bombs in luggage [2], narcotics in cargo containers [3], etc. No sensor or instrument is perfect; most are markedly flawed. But we find that most instruments are nevertheless valuable hit-rate enhancers for the operator/inspector who believes in one enough to learn how to use it and how to interpret its output.

Our concept is to use mobile robots and computer aided signal understanding to replicate and improve this capability by:

- deploying existing instruments using robots that operate at skill level of expert inspectors;

- optimizing instrument sensitivities for deployment by robots, e.g., for methods using penetrating radiation, increasing source strength for improved detection while decreasing operator exposure by robotic deployment;

- augmenting the primary detection modality with complementary sensing 
technologies that, via sensor fusion methods, increase overall system sensitivity and provide a high degree of disambiguation, thereby reducing the false alarm rate;

- providing a picture- and graphics-oriented interface through which the inspectors remotely supervise the robots and instruments;

- providing database and statistical tools to assist the inspectors in making decisions about resource deployment, interpretation of instrument responses, and remedial strategies and tactics.

In the remainder of the paper we discuss in detail the early stages of a project in which we are using this conceptual framework to address the specific problem of inspecting aging aircraft for radial cracks around rivets and for delamination of adhesive bonded lap joints.

\section{The Aging Aircraft Problem}

Aircraft skins inflate and deflate with each "cycle" of pressurization on takeoff and depressurization on landing. The resulting stress causes several kinds of damage, primarily radial cracks around rivets and delamination of adhesive bonded lap joints. The tendency to delaminate is exacerbated by corrosion [4]. The problem of cycling-induced skin failure in aging aircraft first received international attention with the near-loss of Aloha Airlines Flight 243 on April 28, 1988. In the Hawaiian Islands many short flights and the moist tropical climate create a "worst case" situation.

The aging aircraft problem is addressed by airlines, with the assistance of aircraft manufacturers and the FAA, through periodic inspection of known problem areas on each aircraft type. About $90 \%$ of skin inspection activity is visual (by inspectors trained for the task), about $9 \%$ instrumented using eddy current probes, and the remainder by other instrumented methods such as ultrasonic probes. X-ray and other penetrating radiation methods are used to find structural fatigue cracks (related more to flying hours than cycles), but not typically for skin inspection.

Our program, under FAA Aging Aircraft Research Program sponsorship since mid-1991, focuses in its first phases on automation as an aid to the aircraft inspectors who use eddy current probes. We will use machine vision to aid probe placement and robot navigation, and opportunistically to find dents, lightning strikes etc, but computer vision aids to visual inspection are not a formal component of the first phase of the program. The role of computer vision as an inspection (as well as guidance) modality is likely to increase in later phases of the program.

\section{Potential Approaches}

We considered many approaches to automation-assisted eddy current probe deployment, with three main variants: the "car wash", the "cherry picker", and the "window washer". The "car wash" approach envisages a permanent structure through which the airplane is towed for inspection. JAL is experimenting with this approach. But conversations with US commercial airline operators indicate that for operational and economic reasons it is not acceptable to them. The "cherry picker" approach envisages a mobile platform that carries an arm and probe manipulator to aircraft panels needing inspection. A similar approach is in use in the US military for neutron beam and X-ray inspection of fighter planes. But it too is said by the airline operators to be unworkable in the crowded hangar environments of US commercial transport. The "window washer" approach, squat mobile robots under 1 meter 
in length and under $15 \mathrm{~kg}$ in weight, crawling unobtrusively over the skin surface, is an alternative that is acceptable to the airline operators. Small robots also are acceptable to the human inspectors, who see them as tools among others in their kits rather than as competitors for their jobs. Thus we adopt the "window washer" approach. As a development project it is probably the most difficult approach, but as a research project it is probably the most interesting approach. The concept is illustrated by the sketch in Figure 1.

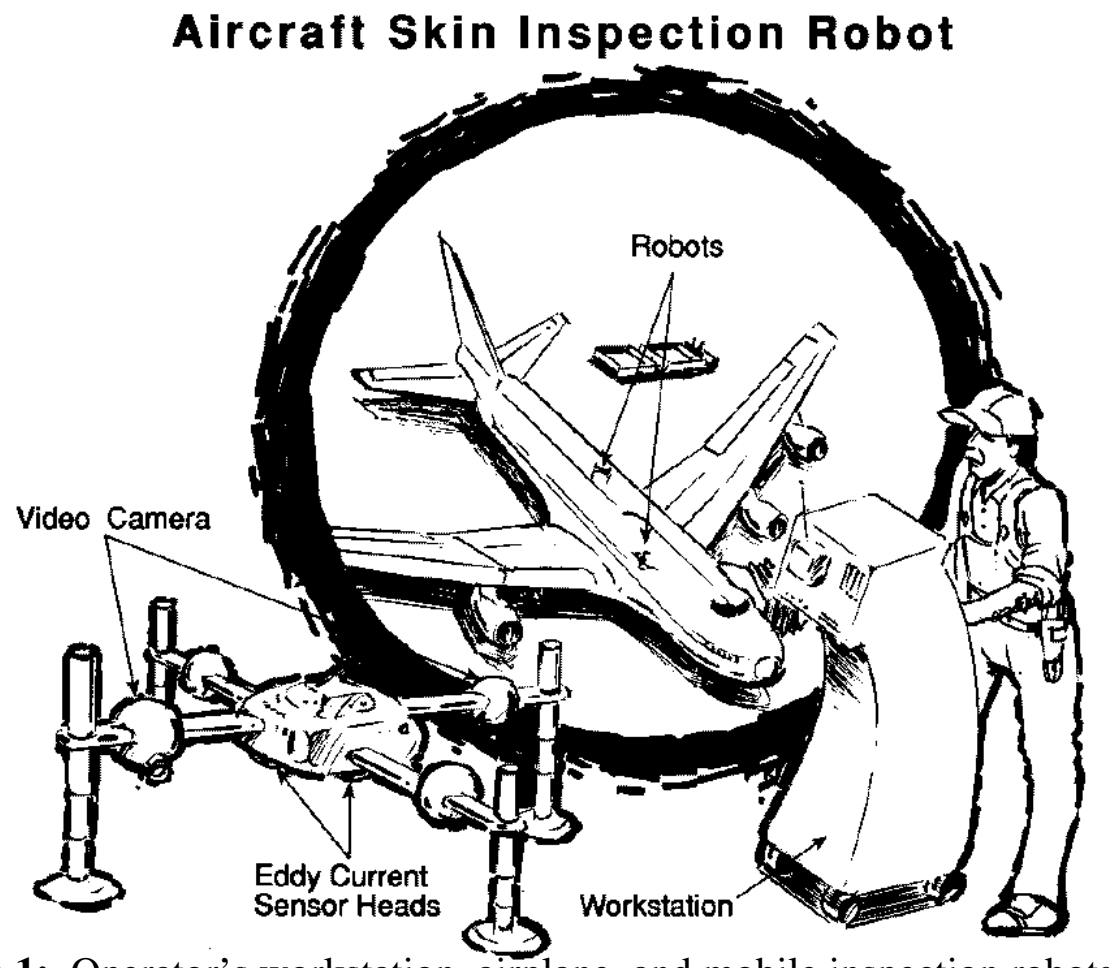

Figure 1: Operator's workstation, airplane, and mobile inspection robots.

\section{Mobile Robot Design}

Most of the skin panels that we expect to inspect are cylindrical fuselage surfaces with "horizontal" and "vertical" rows of rivets. [We use "horizontal" to mean "approximately parallel to the long horizontal axis of the airplane from nose to tail" and we use "vertical" to mean "approximately circumferential around the fuselage."] The inspection task is to move an eddy current probe along a row of rivets that is known (by experience with other aircraft of the same type or by stress failure modeling) to be susceptible to developing cracks, then to move to another (perhaps contiguous) susceptible region and perform another scan. There is typically more horizontal than vertical length that requires inspection. Thus a robot that moves along horizontal lines gracefully, up and down vertical lines adequately, and in random directions perhaps somewhat haltingly is acceptable if compromises in overall grace confer advantages in horizontal inspection capability. With this perspective we have designed the "cruciform" robot depicted in Figure 2. We call the first prototype ANDI, for Automated NonDestructive Inspector. 
ANDI has one long member, the "spine", which will usually be horizontal. It has one suction cup foot at the "head" and two suction cup feet on a short cross member at the "tail." [The designations head and tail are for our convenience in talking about the machine; manipulation and mobility capabilities will both be nearly identical in either direction.] ANDI has two short cross members, the "bridges," which will usually be vertical, each also with suction cup feet at the ends. Each suction cup is mounted on a pneumatic actuator, so ANDI can be supported either by the three feet on the spine or by the four feet on the two bridges.

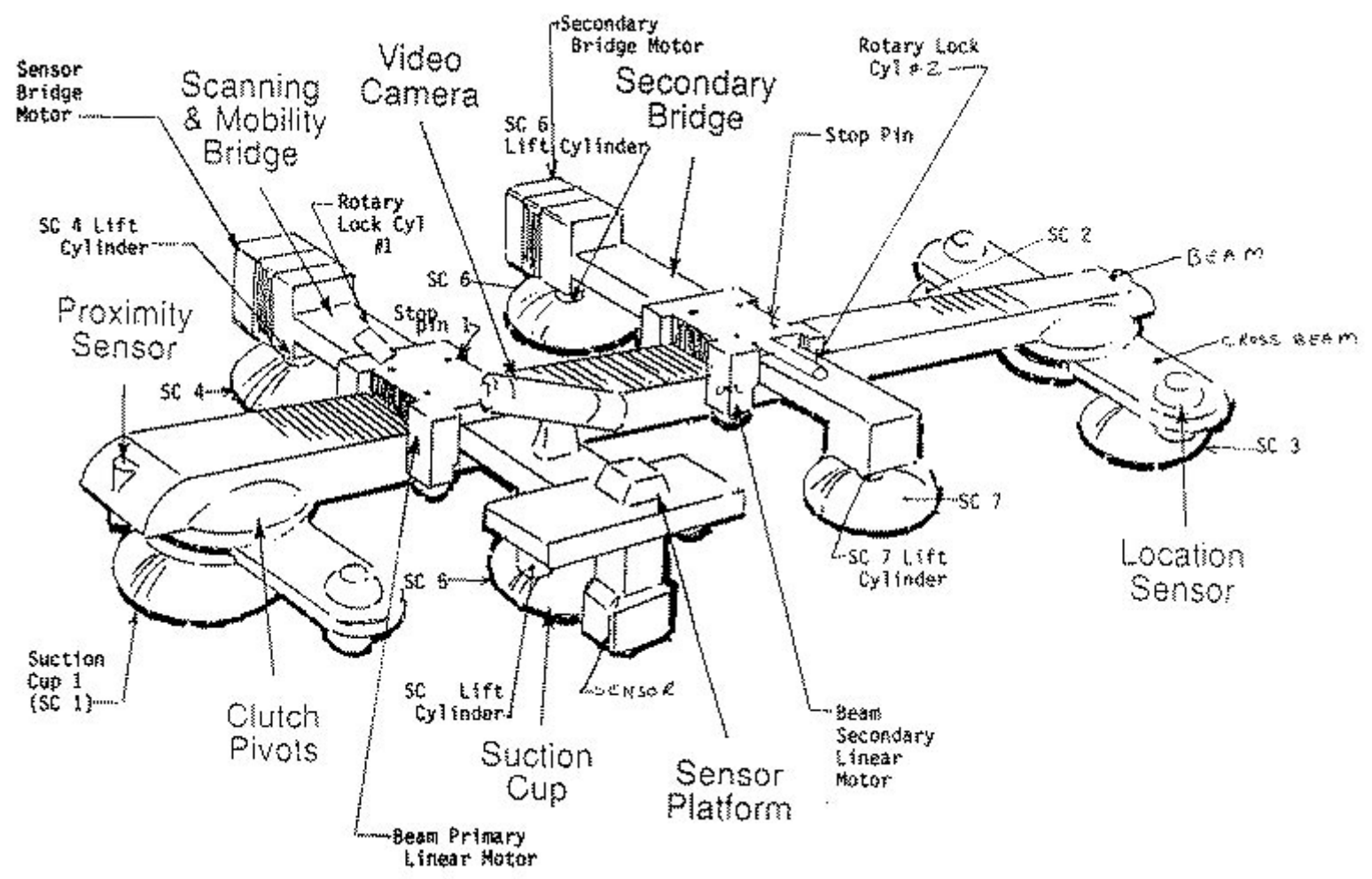

Figure 2: Sketch of cruciform robot design.

The bridges carry and manipulate the sensors ("scanning") and also provide mobility ("walking"). During scanning the spine is lowered, the bridge feet are raised, and the bridges are driven independently back and forth along the spine as required by the inspection protocol. During walking the bridges are driven in parallel by identical message streams, which makes it appear to a naive observer that they are joined by a rigid mechanical link.

To perform a scan, the spine feet lock onto the aircraft skin, and at least one of the bridges manipulates a sensor, normally horizontally, and when necessary vertically. The sensor probe is raised and lowered by a pneumatic actuator that maintains a constant contact force against the aircraft skin. The second bridge can be locked to the skin for additional stability, or it can deploy another sensor (perhaps with a complementary sensing modality), or it can be idle.

To walk, the bridges lock their feet to the fuselage, the spine releases its feet, and driving the bridges backward forces the spine to move forward, and vice versa. When the spine is as far forward as possible, the spine is lowered, the bridge feet are raised, and the bridges are driven forward. The forward motion of the bridges may include scanning, if ANDI is 
moving over a part of the fuselage that requires inspection, or it may simply be part of the gait ANDI uses to move between different areas that require inspection.

A similar sequence, with the bridges driven perpendicular to the spine, is used to walk vertically, and where necessary, to scan vertically.

The joints between the bridges and the spines have a rotary degree of freedom, normally locked at 90 degrees, but which can be unlocked to allow skew motion to accommodate situations where scan paths are neither strictly horizontal or nor strictly vertical, e.g., around aircraft doors.

The bridges are driven along the spine by linear stepper motors, and perpendicular to the spine by zero-backlash lead screws. The $20 \mathrm{kHz}$ oscillator that drives the linear stepper motors is a potential source of interference with the eddy current sensors; however experiments with our motors and sensors indicate that the sensor pickup circuit has sufficiently narrow bandwidth ("high Q") that there is no detectable interference as long as the sensor operating frequency differs from $20 \mathrm{kHz}$ by a few tenths of a $\mathrm{kHz}$.

In addition to electric power and signal lines, compressed air is needed to operate air bearings on the linear motors, pneumatic cylinders that power the up-down motion of the feet, and the ejectors that create (by a "venturi" effect) the vacuum under the suction cups. System compressed air consumption for all these functions is comparable to the consumption of a single air-operated hand tool of the type commonly used by aircraft mechanics.

\section{Eddy Current Sensing}

The eddy current method uses a transmitting and a receiving coil (which may be physically one) coupled electromagnetically through the metal under inspection. Anomalies in the impedance that characterizes the coupling indicate cracks, corrosion thinning, and other flaws. Inspectors are generally given physical standards (created by inspection supervisors) that exemplify the expected flaws via the impedance vs probe position traces displayed by the eddy current instruments. Based on comparison of inspection data with standards data the inspectors report exceptions. Automatically alarming conservatively on apparent exception, and asking the inspector to decide whether or not repair is needed, is straightforward provided the desired path can be followed with adequate accuracy.

Modern eddy current systems already alarm on readings outside setable ranges. In early program stages we will use these alarms to alert the human inspector and mark the area that triggered the alarm, e.g., using a grease pencil or washable paint dab. In later program stages the built-in simple thresholding alarm triggers will be replaced with pattern recognition algorithms or hybrid signature extraction and rule-based decision making systems such as those that have been found useful in automated eddy current inspection of pipe in nuclear power generating facilities [5].

We are planning to achieve the required positional accuracy by high precision deadreckoning over short distances from multiple landmarks: the rivets themselves, each of which is individually identifiable in the aircraft design database. ${ }^{1}$ Small corrections will be made using the vision system (described below) and the eddy current signals themselves, which indicate not only flaws, but also departure of the actual scan line from an ideal scan line across the horizontal diameter of each rivet.

\footnotetext{
${ }^{1}$ In principle these databases exist for all aircraft. In practice they are still on paper for all but the latest generation of aircraft, e.g., the Boeing 777, which are not yet "aging". Thus in practice we will be forced to include some database populating in our program.
} 


\section{Vision System}

The vision system under design anticipates four cameras: one on the eddy current probe carrier for close-up inspection with a field-of-view of one rivet, two fore and aft on the spine for alignment of the robot with lines of rivets or lap joints with a field-of-view of a few tens of centimeters, and one mounted high, capable of pan and tilt, with a wide fieldof-view shared by proprioception and navigation systems. Design issues relating to the realworld environment include dealing with uncontrolled lighting, eliminating (or perhaps exploiting) specular reflections, and locating metal landmarks on a metal background. We anticipate using a hybrid image understanding system with traditional algorithms for feature enhancement and neural networks for interpretation. The image understanding system is now being prototyped using independently collected airplane skin images for evaluating feature enhancement algorithms and neural net architectures.

In the early demonstration phases the vision system will be used for aiding automatic alignment of the eddy current probe, for aiding automatic alignment of the scanning and walking motions with rivet or lap joint lines, and for aiding the operator via monitors at the inspector's workstation. In a later phase of the program we anticipate adding long range navigation and collision avoidance to the vision system's capabilities, and in a more distant phase it may be possible to use computer vision for strictly visual aircraft inspection tasks. We caution that machine vision for automated skin detection may prove to be a very difficult problem.

\section{Interface and Controls}

The aircraft inspector's interface to the eddy current sensor, vision system, and robot control system is via a menu system implemented on an 80486/DOS PC. We anticipate adding another similar PC to support the vision system's image acquisition and processing. The menu system, when completed, will allow the inspector to describe the requirements of an inspection in high level terms: walk to a specified location, initialize the eddy current sensor location and electrical setup, scan along a specified vector, display scan data in specified format, etc. As automatic aids to exception alarming and more sophisticated data analysis are implemented they will provide increasingly sophisticated forms of advice to the inspector. At first this advice will be no more complicated than marking the location of an out-of-bounds indication directly on the skin and by a cursor superimposed on the appropriate camera image. Later it will involve interactions with both the inspector and the database to maximize the probability of flaw detection, minimize the probability of false alarms, analyse crack growth trends, etc.

The major system control functions are implemented via RS-232 communication lines transmitting command and status messages between the computer's serial ports and the linear stepper motor controllers and the lead screw controllers. The pneumatic components are controlled by solenoid valves operated by digital I/O lines on a multipurpose (analog, digital, time, etc) card on the PC bus. This card handles input and output for a variety of additional sensors and actuators, e.g., suction cup vacuum control via solenoid valves and monitoring the vacuum to assure proper sealing via differential pressure switches. The latter action is especially important to assure that ANDI does not fall when walking on the sides or belly of the fuselage. 


\section{Recording and Archiving}

Aircraft skin inspections are now pass/fail. There is no record of cracks or corrosion thinning below the official reporting threshold. This practice is not regarded as a hazard because the thresholds have substantial safety margins, and there are good flaw growth models. We nevertheless feel, and the FAA and airline operators agree, that the predictive capabilities that will follow database representation of quantitative inspection results will facilitate maintenance scheduling and potentially increase safety. Thus database representation, with an architecture open to access from multiple potential inspection and maintenance locations and tools for trend analysis and improved statistical predictions, is an integral part of our program.

In this context we will also compile a design exception database on each aircraft. Here we will record special modifications, repair patches, plated regions, regions with oversize replacement rivets, etc. These exceptions will be useful aids to navigation as well as to maintenance.

\section{Status and Plans}

An airplane panel has been procured for laboratory tests. ANDI is undergoing manually controlled scanning and walking tests, component characterization, and concept refinement concurrently with building of the control, sensor, and database systems. Figure 3 shows ANDI during these early tests. At the conference we will present data on early performance in the laboratory, plans for testing on aircraft in maintenance hangars, and future plans and prospects.

\section{Acknowledgements}

The system architecture was first articulated by author MWS during discussions with Alberto Guzman concerning concepts for detection of contraband in cargo containers. The system implementation for the aging aircraft inspection application was developed in close collaboration with author WMK. The mechanical, control, sensor, and human interface designs and implementations for the ANDI robot system were created in collaboration with Chris Carroll, Ian Davis, Alan Guisewite, John Hudak, and Court Wolfe. Portions of this work were funded by the FAA Aging Aircraft Research Program under Grant G0319014. 


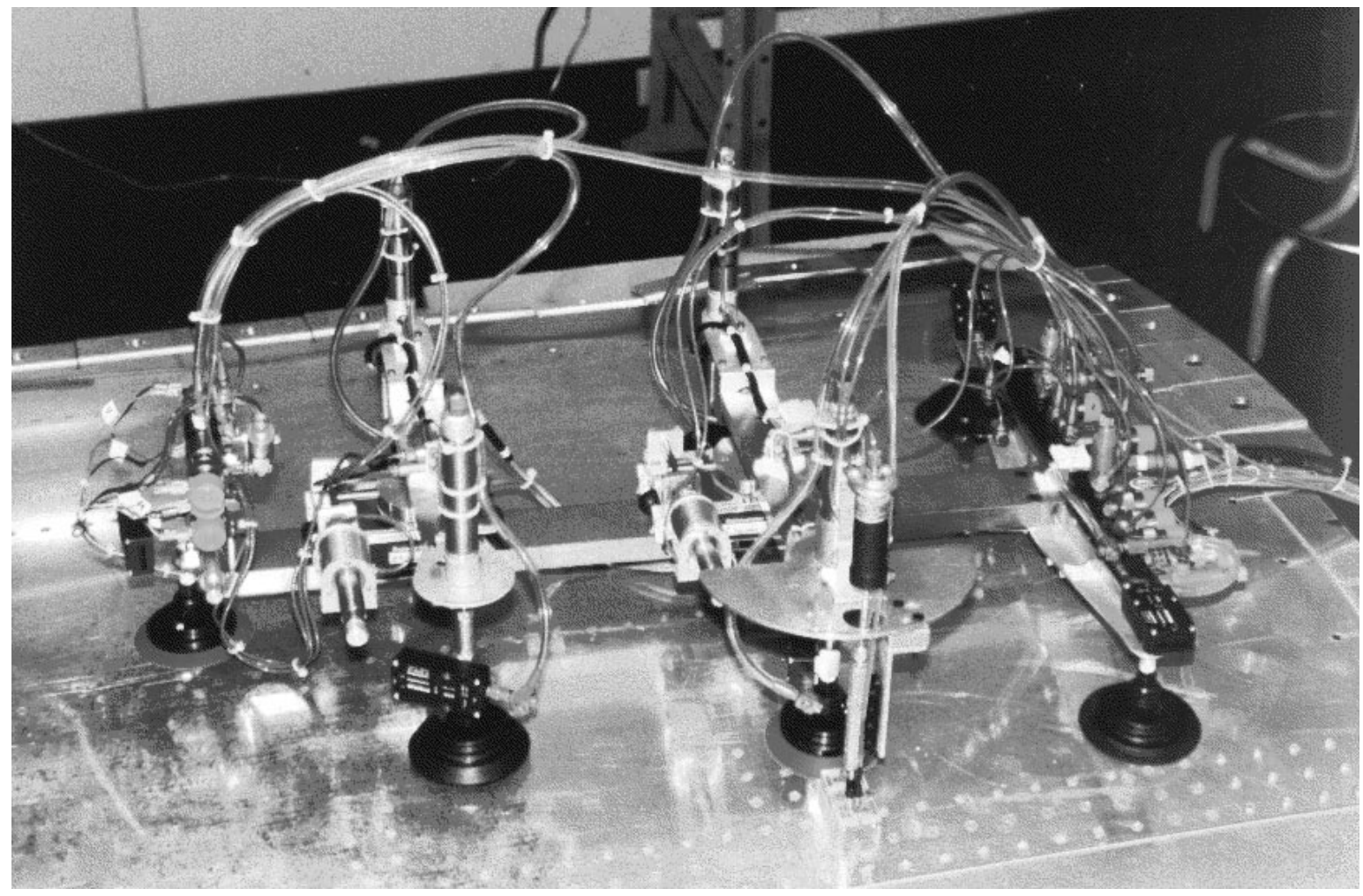

Figure 3: ANDI undergoing early tests on an airplane panel.

\section{References}

1. W. M. Kaufman, M. W. Siegel, C. J. Alberts, "Robot for Automation of Aircraft Skin Inspection', Proceedings of the International Workshop on Inspection and Evaluation of Aging Aircraft, Benham Bahr,ed., Federal Aviation Administration (FAA) hosted by Sandia National Laboratories, Albuquerque NM, May 1992, pp. VII 13-18.

2. M. W. Siegel, A. M. Guzman, W. M. Kaufman, "Robotics Systems for Deployment of Explosive Detection Sensors and Instruments', Proceedings of the First International Symposium on Explosive Detection Technology, Siraj M. Kahn,ed., FAA Technical Center, Federal Aviation Administration (FAA), Atlantic City International Airport, NJ 08405, February 1992, pp. 873-9, (conference held November 13-15, 1991.)

3. M. W. Siegel, A. M. Guzman, and W. M. Kaufman, "Robotic Systems for Deploying Sensors to Detect Contraband in Cargo", Proceedings of the Washington DC Meeting, Albert E. Brandenstein ,ed., Office of National Drug Control Policy and National Institute of Justice, Executive Office of the President, Washington DC 20500, October 1992, pp. 345-52.

4. 1991 International Conference on Aging Aircraft and Structural Airworthiness, Federal Aviation Administration (FAA) and NASA, FAA and NASA, Charles E. Harris, Washington, DC, Nov 1991.

5. Soon-Ju Kang, Nam-Seok Park, and Yong-Rae Kwon, “A Hybrid Expert System for Eddy Current based Inspection of Steam Generator Tubes in Nuclear Power Plant', Third Symposium on Expert Systems Application to Power Systems (TokyoKobe, JAPAN), Korea Atomic Energy Research Institute and Korea Advanced 
Institute of Science and Technology, Dajeon City and Seoul, Korea, Apr 1991, pp. 144-151. 